\title{
Un texte opaque et son interprétation sociohistorique : « Barbare
} » de Rimbaud

In: Romantisme, 1983, n³9. pp. 127-142.

Citer ce document / Cite this document :

Wetzel Hermann. Un texte opaque et son interprétation sociohistorique : « Barbare » de Rimbaud. In: Romantisme, $1983, \mathrm{n}^{\circ} 39$. pp. 127-142.

doi : $10.3406 /$ roman.1983.4668

http://www.persee.fr/web/revues/home/prescript/article/roman_0048-8593_1983_num_13_39_4668 
Un texte opaque et son interprétation socio-historique : "Barbare" de Rimbaud

I - La poésie ne fait pas partie du champ préféré des recherches dites socio-historiques. Le théâtre classique, la littérature du siècle des lumières, le roman réaliste s'y prêtent mieux à première vue, le reflet de la société et de son histoire y semble plus immédiat. Leurs héros expriment des opinions sur des personnages et sur des événements politiques et sociaux; pour motiver l'action des personnages du théâtre et du roman, il faut l'étoffer de nombreux fragments de réalité qui permettent une relation plus ou moins directe avec l'époque.

Or, la poésie moderne qui a dépassé la "prose rimée" (1) se refuse à un tel accès. L'écart entre la réalité socio-historique et le "monde" des oeuvres poétiques (qu'on pense à la théorie de l'art pour l'art) devient de plus en plus infranchissable. S'inspirant par exemple de l'article fondamental de Claude Pichois sur Baudelaire en 1847, "petits essais de sociologie littéraire" (2), les chercheurs qui tentent quandmême une approche socio-historique s'appuient de préférence sur des textes non-poétiques (articles, lettres, journaux intimes, etc.) qui traitent directement, et d'une façon discursive et conceptuelle, des problèmes de leur temps, ou sur des poèmes qui renferment quelque profession de foi philosophique. Rares sont les essais, et non pas toujours satisfaisants, de tenter une interprétation socio-historique de poèmes qui, par leur contenu manifeste, n'ont rien à faire avec des

(1) Le mot est de Rimbaud (Lettre à P. Demeny du 15-5-71) : "De la Grèce au mouvement romantique - moyen-âge - il y a des lettrés, des versificateurs. D'Ennius à Théroldus, de Théroldus à Casimir Delavigne, tout est prose rimée, un jeu, avachissement et gloire d'innombrables générations idiotes." Voir R. Barthes. "Y-a-t-il une écriture poétique ?", dans Le degré zéro de l'écriture, Coll. "Points", éd. du Seuil, 1972, p. 33-40; ici p. 33 : “La poésie classique n'était sentie que comme une variation ornementale de la Prose, le fruit d'un art (c'est-à-dire d'une technique), jamais comme un langage différent ou comme le produit d'une sensibilité particulière. Toute poésie n est alors que l'équation décorative, allusive ou chargée, d'une prose virtuelle [...]"'

(2) Dans Baudelaire, Etudes et témoignages, Neuchâtel 21967 , p. $95-121$; depuis, l'Allemagne a connu une véritable découverte d'un Baudelaire "révolutionnaire"; voir H. Stenzel, Der historische Ort Baudelaires, München, Fink, 1980. 
événements historiques précis, et qui demanderaient par conséquent une interprétation socio-historique de leur forme, ou mieux, de leur structure (3).

I I - C'est la poésie moderne qui, par son opacité et son "inintelligibilité", a ouvert les yeux sur le fait que les vertus de la fonction poétique du langage dépassent de loin celles d'un discours orné de quelque rime, rythme ou belle image. La "plus-value" (4) poétique n'est que latente dans la poésie classique et dans une grande partie de la poésie romantique : les poètes expriment une opinion, une vision du monde entièrement faite de concepts préalables; ce n'est que dans la poésie moderne que le lecteur, à défaut d'une image "réaliste", d'un modèle reconnaissable au sein de sa propre "réalité" idéologique, est forcé - s'il ne se borne pas à hausser les épaules - de reconstruire, à partir d'éléments disparates et de débris provenant d'anciens modèles courants, un nouveau mode de réalité, plus adéquat, plus "vrai", plus approprié (5).

Le trait fondamental de toute littérature, et tout particulièrement de la poésie moderne, c'est son caractère métaphorique. D'après $P$. Ricoeur et Ju. M. Lotman, je vois dans la $m$ é $t$ a $p h$ or e et dans le $\bmod \dot{e} l e$, cette grande métaphore qu'est le poème entier, la clé de voûte de toute théorie et de toute pratique de l'interprétation. Ces deux concepts, la métaphore et le modèle, qui ne sont que la manifestation du même procédé métaphorique à deux niveaux de la même échelle, permettent de fixer et d'analyser le point de médiatisation entre la réalité et le langage, entre l'idéologie et la littérature à partir de phénomènes linguistiques et littéraires.

I I A - Le propre de la mét aphore n'est pas de substituer un mot à un autre, plus pittoresque ou plus décoratif, mais de mélanger et de faire interagir deux aires sémantiques incompatibles jusqu'alors dans le code linguistique dominant - interaction qui fait surgir un sens nouveau - quelque chose d'inconnu, d'inexprimable par le langage courant. Du point de vue linguistique, il n'y a pas simple substitution paradigmatique (6), mais création d'une équivalence sémantique, qui devrait se situer sur l'axe paradigmatique (sélection) par un acte de combinaison sur l'axe syntagmatique. C'est ainsi que

(3) Ces recherches sont en général plutôt thématiques que structurales. On connaît l'exemple de W. Benjamin où l'intuition géniale dépasse l'analyse; voir la lettre de Th. W. Adorno à W. Benjamin du 10-11-1938. W. Fietkau, Schanengesang auf 1848, Reinbek bei Hamburg, Rowohlt, 1978, poursuit cette ligne en déployant un large contexte, littéraire, politique, architectural et philosophique, malheureusement sur la base d'un seul poème (Le Cygne).

(4) P. Ricoeur, La Métaphore vive, éd. du Seuil, 1975, p. 315.

(5) Ju. M. Lotman, Die Struktur literarischer Texte, München, Fink, 1972, p. $412-413$.

(6) R. Jakobson: “Deux aspects du langage et deux types d'aphasie", dans Essais de linguistique générale, Paris, éd. de Minuit, 1963; en plus : P. Ricoeur, ouvr. cit., p. 252. 
la métaphore devient le prototype du célèbre principe d'équivalence de R. Jakobson (7) au niveau sémantique. D'éléments dissemblables et incompatibles, surgit le semblable par simple juxtaposition syntagmatique. Comme la rime, qui par une équivalence de sons suggère inévitablement une équivalence sémantique (8), une combinaison de deux (teneur plus véhicule) (9) ou de plusieurs isotopies, transgresse les lois métonymiques de contiguïté d'un lexique codifié, et fait voir "le même dans le différent", "le semblable" (10) ou mieux "du nouveau". En ce qui concerne le système de valeurs idéologiques qui est intimement lié au système conceptuel du lexique, la métaphore est une "méprise catégoriale (11)." Une seule de ces méprises change tout le système sémantique et par conséquent toute une idéologie (12), tandis qu'une mét on y mie ne produit qu'un changement à l'intérieur du système de contiguïté admis par la communauté linguistique.

Prenons un exemple qui nous rapproche de Barbare: on peut lire dans Le Peuple de J. Michelet (13), et Rimbaud l'a probablement lue aussi, la phrase suivante :

"Souvent aujourd'hui, l'on compare l'ascension du peuple, son progrès, à l'invasion des Barbares. Le mot me plaît, je l'accepte [...]"

Sans nous attarder sur la différence entre comparaison et métaphore, on peut dire que la phrase reste, à première vue, dans le cadre conceptuel du langage courant qui utilise "barbare" comme synonyme de "sauvage", "brute" et "féroce", et en même temps dans le cadre d'une idéologie nobiliaire-bourgeoise qui charrie au milieu du

(7) R. Jakobson, "Linguistique et poétique", dans Essais, p. 209 - 248.

(8) Ibid., p. 235 : "Bref, l'équivalence des sons, projetée sur la séquence comme son principe constitutif, implique inévitablement l'équivalence sémantique [...]". (9) C'est la terminologie de I. A. Richards, The Philosophy of Rhetoric, Oxford, Oxford University Press, 1936.

(10) P. Ricoeur, ouvr. cit., p. 249 - 250 : “[...] le "semblable" est aperçu en dépit de la différence, malgré la contradiction. La ressemblance est alors la catégorie logique correspondant à l'opération prédicative dans laquelle le "rendre proche " rencontre la résistance du "être éloigné"; autrement dit, la métaphore montre le travail de la ressemblance [...] le "même" et le "différent" ne sont pas simplement mélés, mais demeurent opposés."

(11) Expression de G. Ryle The Concept of Mind, London, Hutchinson, 1949, p. 8, citée chez P. Ricoeur, ouvr. cit. p. 250 . Ricoeur résume (p. 251) : 'L'idée de méprise catégoriale conduit près du but. Ne peut-on pas dire que la stratégie de langage à l'oeuvre dans la métaphore consiste à oblitérer les frontières logiques et établies, en vue de faire apparaître de nouvelles ressemblances que la classification antérieure empêchait d'apercevoir ? Autrement dit, le pouvoir de la métaphore serait de briser une catégorisation antérieure, afin d'établir de nouvelles frontières logiques sur les ruines des précédentes."

(12) H. Weinrich, "Semantik des Metapher", dans Folia linguistica 1, 1967, p. 12: "Ändert sich die Bedeutung eines Wortes, so ändern sich die Bedeutunngen aller Wörter." ["Si un mot change de sens, tous les autres mots en sont concernés." ].

(13) J. Michelet, Le Peuple, éd. P. Viallaneix, Paris, Flammarion, 1974, p. 72. 
XIXème siècle le préjudice d'un peuple inculte, brutal et destructeur (14). Mais Michelet continue :

“Barbares! Oui, c'est-à-dire pleins d'une sève nouvelle, vivante et rajeunissante. Barbares, c'est-à-dire voyageurs en marche vers la Rome de l'avenir [...] Nous avons, nous autres Barbares, un avantage naturel; si les classes supérieures ont la culture, nous avons bien plus de chaleur vitale."

Michelet a ressuscité une métaphore morte qui a été lexicalisée comme mot à signification nettement négative, en lui assignant un contexte exclusivement positif. Au lieu de ne retenir de l'invasion des barbares à la fin de l'Empire romain, que le côté destructeur, Michelet n'en retient que l'aspect progressif, rénovateur: "barbare" est devenu une métaphore de valeur positive. Michelet - quoi qu'en disent ses détracteurs - non pas poète, mais historien au langage conceptuel, substitue une signification positive à la signification négative. Pour y arriver, il est forcé d'arrêter, par une paraphrase conceptuelle, le procès métaphorique qui a été entamé par la vivification de l'image historique "Invasion des Barbares" et qui contient deux éléments, le négatif et le positif ensemble. (On verra quel usage différent en fera Rimbaud en travaillant sur le même mot.) Ici, c'est surtout le côté idéologique de ce changement métaphorique du sens d'un seul mot qui nous intéresse, changement seulement pensable au sein de tout un nouveau modèle de la société française au XIXème siècle.

I I B - Un $\bmod \dot{e} l e$ poétique est une métaphore soutenue (15), s'il ne contient que deux isotopies, une sorte de métaphore filée. Comme pour la métaphore le sens "propre" du modèle reste temporairement suspendu jusqu'à ce que le nouveau sens "impropre" (la référence secondaire) soit établi par le procès métaphorique. La prédominance de la fonction poétique du langage n'abolit pas les autres fonctions, par exemple la fonction référentielle; au contraire, elle ne fait que l'intensifier (16) en la scindant en deux : le monde de l'oeuvre est doublé par une réalité dont ce monde de l'ouvre est le modèle. Aucune ouvre, aucun modèle littéraire n'est une description "propre" reflétant la réalité, mais seulement son modèle (entre autres modèles possibles). Quelquefois, dans des ouvres dites réalistes, cette différence ontologique entre réalité et modèle, est cachée ou plutôt moins évidente que dans des oeuvres opaques, par le simple fait que la contiguïté métonymique de l'oeuvre (son réalisme) correspond au modèle du monde fixé et codifié dans le système linguis-

(14) On n'a qu'à lire Les Paysans de Balzac; voir L. Chevalier, Classes laborieuses et Classes dangereuses à Paris, pendant la première moitié du XIXème siècle, Plon, 1978.

(15) M. Black, Models and Metaphors, Ithaca, Cornell University Press, 1962, p. 236 .

(16) Voir R. Kloepfer, Poetik und Linguistik, München, Fink, 1975. 
tique communément admis à un moment historique donné - par conséquent le lecteur identifie par mégarde la réalité et son modèle.

A y voir de près, même les modèles réalistes, en tant que modèles, ne correspondent pas tout à fait à l'idéologie dominante : tantôt ils pèchent par un excès de conformité à ce modèle idéologique (c'est le cas du "Kitsch"), tantôt ces modèles intègrent des éléments de plusieurs idéologies existant, à une même époque, mais pas toutes également admises par l'idéologie plus ou moins officielle, codifiée dans les lois et réalisée dans les institutions. Bref, chaque ouvre littéraire est une redescription (réécriture) plus ou moins différente de modèles déjà existants, littéraires ou non.

I I C - Selon le degré de prédominance de la fonction poétique du langage et surtout du procès métaphorique, on pourrait distinguer grosso modo trois types de poésie. La séquence de ces trois types correspond à peu près aux étapes de leur développement au cours du XIXème siècle, mais ils coexistent toujours, avec de nombreuses transitions, comme possibilité poétiques fondamentales.

Prose rimée: mise en scène décorative d'un discours conceptuel, d'une narration ou d'une description réaliste (idéologique) du monde. La métaphore n'est qu'un ornement entre autres (rime, prosodie, etc.); l'organisation métonymique prédomine.

Modele simple : une image, un modèle réaliste, c'est-à-dire organisé à son intérieur d'une façon métonymique (= véhicule) fonctionne (ou peut fonctionner) globalement comme métaphore (symbole) d'une autre réalité ( = teneur); par exemple, toute sorte de "volaille" (albatros, cygne, aigle) pour symboliser le poète.

Quant à leur dépendance d'un système idéologique préconçu, ces modèles simples peuvent varier d'une soumission presque complète à une idéologie préalable (c'est le cas de l'allégorie) jusqu'à une extrême liberté qui ne permet même pas de trouver une teneur indubitablement identifiable (modèles absolus de L'art pour l'art). Mais à force d'être organisés intérieurement d'une façon métonymique, ces modèles se fondent sur une connivence fondamentale avec les visions du monde déjà existantes et figées dans le système du langage à un moment historique. Leur tendance peut être critique, voire oppositionnelle (V. Hugo) ou évasive (Gautier, Les Parnassiens); ils critiquent au nom de valeurs idéales anciennes (antiques, chrétiennes) toujours en vigueur mais en réalité non pratiquées, ou ils s'évadent dans des pays lointains, sauvages, exotiques ou artificiels, sans atteindre directement les valeurs en vigueur. Bien qu'ils contrastent par leur beauté idéale ou par l'esthétisation du laid avec la réalité vécue, ils ne produisent que des changements à l'intérieur du système de valeurs établi. La notion du Mal n'est pas surmontée par les Fleurs du Mal, elle n'est qu'assumée et non plus refoulée : le système du bien et du mal reste intact (17).

(17) H. Nöding, "Das Blut im Rinnstein, der Lumpensammler und die Schönheit. Themen Baudelaires", dans P. Brockmeier / H. H. Wetzel, éd. Frankreich in seiner Literatur, Bd. 3, Stuttgart, Metzler, 1982, p. 123-170, a souligné l'importance de la formule "faire le Mal, le sachant" pour le satanisme de Baudelaire. 
Pour déchirer la camisole de l'idéologie inhérente à tout langage intelligible (18), car métonymique, il faut pousser le procès métaphorique le plus loin possible; il faut détruire la contiguité métonymique, détruire le "réalisme" du modèle, rendre le texte opaque et incompréhensible, pour pouvoir construire un

Modèle complexe : les deux isotopies hiérarchisées et parallèles des modèles simples (ou la seule isotopie du modèle absolu) sont remplacées par une structure complexe d'isotopies différentes, voire opposées, provenant des débris de modèles du monde antécédents. Chez Rimbaud la phase de destruction de modèles est caractérisée par le fameux "dérèglement" et son correspondant poétique la parodie (19). Pour éviter le chaos, pour garantir le caractère de modèle, et pour permettre au delà de la référence primaire suspendue l'interprétation d'une référence. secondaire, le poète établit une structure qui utilise tous les registres de la fonction poétique du langage. Comme la métaphore, dans la poésie moderne, est promue du rang d'une simple décoration au rang d'un moyen authentique de connaissance, toutes sortes de parallélismes (phonologiques, rythmiques, morphologiques, syntaxiques etc.) sont affranchis de leur fonctionnement plutôt mécanique (dans le meilleur des cas de signification redondante) au sein de poèmes rimés à forme fixe, pour gagner une liberté complète de signifier. Une rime dans un texte non rimé, un rythme dans un texte non-métrique, un alinéa dans un texte non-strophique etc. devient significatif par son exceptionnalité. Cette destruction des règles de la versification traditionnelle faisant place à une nouvelle mise en valeur de leurs procédés primitifs et liée au nouveau sémantique, à une métaphorisation continue, aboutit sous le coup d'une signification généralisée par le principe d'équivalence à une sémantique de la forme.

Le manque d'un contenu de prime abord compréhensible force le lecteur à détruire, lui aussi, ses propres images toutes faites de la réalité, ses certitudes idéologiques, de suivre pas à pas la structuration effectuée par le poète et de créer avec lui à partir de débris des anciens, un nouveau modèle du monde, ou même le modèle d'un nouveau monde.

(18) A. Jouffroy, ("Surréalisme et poésie", dans F. Alquié, Entretiens sur le Surréalisme, Paris / La Haye, Mouton, 1968, p. 130) cite la phrase de A. Artaud "Tout vrai langage est incompréhensible." et continue : "Telle est la vérité que nous n'avons pu encore digérer. Tout le reste vraiment serait littérature, et dans la mesure où elle n'est pas vraie, toute littérature demeure infiniment et désespérément compréhensible. C'est la fatalité des écrivains, fatalité elle aussi interne mais pardonnable, qui les condamne, tôt ou tard, à se placer au centre de la compréhension."

(19) Voyez aussi la place de la parodie dans la théorie des formalistes russes; J. Striedter, Russischer Formalismus, München, Carl Hanser, 1969, p. XXXIX et suiv. et les articles de V Sklovskij, "Der parodistische Roman", ibid. p. 245 et suiv. et de J. Tynjanov, "Dostoevskij und Gogol (Zur der Theorie der Parodie)", ibid. p. 301 et suiv. 
I I I - Rimbaud, et c'est ce qui fait la fascination de son ouvre, a parcouru toutes ces possibilités poétiques en moins de quatre ans (de 1870 à 1874 à peu près) : des premières imitations de la prose rimée romantique, et de son idéologie présocialiste et progressiste ("Soleil et Chair", "Le Forgeron") en passant par les parodies des "Lettres du Voyant" ("Le Coeur supplicié", "Chant de guerre parisien", "Mes Petites amoureuses", "Accroupissements") qui marquent sa distanciation envers les modèles poétiques en vogue, jusqu'aux "Illuminations". Il va même plus loin encore, jusqu'à renoncer à la poésie, sous l'impression du fait que tout en étant "en avant" de la société, tout reste littérature, si l'action ne suit pas.

Le stade des modèles complexes (la poésie "objective" de Rimbaud) est le plus intéressant et le plus innovateur, puisque c'est ici qu'on assiste à une sorte de critique des idéologies par des moyens poétiques authentiques. C'est la destruction des modèles idéologiques existants, et le travail créatif de la construction d'un nouveau modèle qui forcent le lecteur à une méta-noia, à un changement de son point de vue et du contenu de ses concepts, à une nouvelle vision $\mathrm{du}$ monde. Les vertus de la fonction poétique du langage et du procès métaphorique sont poussées jusqu'à l'abandon presque total de l'enchaînement métonymique, c'est-à-dire jusqu'au seuil de l'incommunicabilité.

Les modèles complexes correspondent à une attitude entre la sécurité d'une idéologie toute faite qui permet une description réaliste et critique de la réalité (par ex. "Le Mal") et la résignation de changer n'importe quoi par voie de littérature ("Conte"). Rimbaud avait atteint ce stade à une époque où, après la défaite de la Commune de Paris, et en face d'une Troisième République nettement restauratrice à ses débuts, il avait perdu la foi dans la loi du progrès et dans une efficacité directe de la poésie sur la politique, sans avoir (encore) pour autant abandonné l'idée d'une poésie qui aiderait l'homme à connaître mieux le monde et qui penserait le futur. Cette place de la poésie entre critique et utopie d'un côté et résignation de l'autre, reste d'une ardente actualité.

I V - Notre interprétation socio-historique d'un texte opaque tel que "Barbare" de Rimbaud (20) parcourt trois étapes : nous essayons d'abord d'analyser les traits significatifs de la structure du texte, de les synthétiser ensuite et de construire ainsi le modèle propre, la vision

(20) Voir aussi les témoins (en partie involontaires) de cette opacité : A. Adam (éd. A. Rimbaud, Oeuvres complètes, "Bibl. de la Pléiade", Gallimard, 1972, p. 1004-1005), S. Bernard (éd. Rimbaud, Oeuvres, Class. Garnier, 1960, p. $518-$ 519), A. Py (éd. A. Rimbaud, Illuminations, TLF 147, Genève / Paris, Droz, 1969 , p. 178 - 181), R. Kloepfer / U. Oomen, Sprachliche Konstituenten moderner Dichtung. Entwurf einer deskriptiven Poetik-Rimbaud - Bad Homburg v.d.H. Athenäum, 1970, p. 74 - 76 et A. Kittang, Discours etJeu, Bergen / Grenoble, (P.U.G.), 1975 , p. $298-304$. 
spécifique de Rimbaud sur tel détail du monde contemporain. Cette construction du modèle du "Barbare" rimbaldien ne peut s'effectuer que sur le fond des autres images et en concurrence avec elles, images utilisant un même concept faisant partie des autres idéologies contemporaines, bref, en situant le modèle dans l'histoire.

\section{B A R B A R E}

I Bien après les jours et les saisons et les êtres et les pays,

I I Le pavillon en viande saignante sur la soie des mers et des fleurs arctiques; (elles n'existent pas.)

I I I Remis des vieilles fanfares d'héroïsme-qui nous attaquent encore le coeur et la tête - loin des anciens assasins -

I V Oh! Le pavillon en viande saignante sur la soie des mers et des fleurs arctiques; (elles n'existent pas )

V Douceurs!

V I Les brasiers, pleuvant aux rafales de givre, - Douceurs! - les feux à la pluie du vent de diamants jetée par le coeur terrestre éternellement carbonisé pour nous. - O monde ! -

VII (Loin des vieilles retraites et des vieilles flammes, qu'on entend, qu'on sent)

VIII Les brasiers et les écumes. La musique, virement des gouffres et choc des glaçons aux astres.

IX O Douceurs, ô monde, ô musique! Et là, les formes les sueurs, les chevelures et les yeux, flottant. Et les larmes blanches, bouillantes, - ô douceurs ! - et la voix féminine arrivée au fond des volcans et des grottes arctiques.

$X \quad$ : Le pavillon ...

La macrostructure de Barbare s'appuie sur une espèce de refrain :

"Le pavillon en viande saignante sur la soie des mers et des fleurs arctiques; (elles n'existent pas.)"

Mais ce refrain ne revient ni régulièrement ni sous une forme identique. Le texte s'articule sur les trois seules répétitions (sur dix paragraphes, refrains inclus) et le refrain lui-même est soumis à un certain développement. Sa deuxième apparition est introduite par un "oh !" admiratif qui, avec l'exclamation "Douceurs!" du paragraphe suivant, signale un développement heureux. La troisième fois, le refrain ne réapparaît plus dans toute sa longueur, mais seuls les deux premiers mots en sont répétés en guise de rappel : "Le pavillon ...". Ce n'est pas pour faire économie d'encre d'imprimerie, mais ce court rappel indique qu'après cette longue partie du texte qui n'est pas interrompue 
par le refrain, sa répétition complète n'est dorénavant plus nécessaire. Tandis que les paragraphes I et III sont respectivement suivis du refrain (II et IV) les paragraphes V - IV restent sans interruption. La distribution des refrains fait alors ressortir la bipartition du texte (I IV et V - X). En plus, et pour souligner cette bipartition, le paragraphe central, qui ouvre la deuxième partie (V) est mis en relief par la brièveté d'un seul terme (plus tard répété): "Douceurs!". Les deux parties se distinguent en outre par leur ordre temporel, la construction des phrases et le rythme des phrases.

Les paragraphes I et III contiennent plusieurs indications temporelles (bien après, remis des, encore, loin de, vieilles, anciens) qui relèguent ce qui suit dans un avenir lointain, comme le résultat d'une victoire et d'une rupture avec un mauvais état des choses présent ("remis de"). Au milieu de la deuxième partie (VII), cette relation temporelle est reprise avec des mots presque identiques ("loin", deux fois "vieilles"). Mais ce paragraphe n'est qu'une parenthèse dans le rythme entraînant des paragraphes adjacents, et ne fait que rappeler l'état présent ("on entend", "on sent") antérieur à la vision poétique des autres paragraphes (V plus VI, VIII plus IX). Le texte contient encore une autre parenthèse à la fin du refrain qui, elle aussi, renferme un verbe au présent ("Elles n'existent pas."). Par cette précision, le contenu précédent du refrain est repoussé au futur, à l'étape visionnaire; le refrain est par conséquent l'endroit qui réunit les deux temps (le futur et le présent, la vision qualifiée d'irréelle) et qui, par sa reprise, encadre les deux parties du texte.

La construction des phrases est aussi différente dans ceux des paragraphes qui parlent du présent, et dans ceux qui parlent de la vision du futur. Les premiers contiennent les seules formes verbales déclinées et des propositions subordonnées relatives, tandis que les secondes n'utilisent que des participes intemporels et impersonnels ou renoncent tout à fait aux verbes et aux phrases complexes. Les deux parties du texte ne se scindent cependant pas en deux séquences incohérentes, mais elles s'engrènent l'une dans l'autre, donnant à la deuxième partie, la vision, une nette prépondérance :

lère partie (état présent)

I

II (Refrain)

III

IV (refrain $=$ II')

$\mathrm{V}$

VI

2ème partie (vision)

$$
\begin{aligned}
& \text { (VII) } \\
& \text { VIII } \\
& \text { IX (Début du refrain = II') }
\end{aligned}
$$

Le refrain est pour ainsi dire la clé de la structure du texte et de sa signification. Sa fascination poétique se fonde d'un côté sur ses 
métaphores qui combinent des termes incompatibles, et de l'autre sur l'euphonie d'un enchaînement par altération $(/ \mathrm{v} /, / \mathrm{s} /)$ et par assonance $(/ \mathrm{o} /, / \mathrm{a} /)$ :

"Le pavillon en viande saignante sur la soie des mers et des fleurs arctiques; (elles n'existent pas.)"

Les incompatibilités du niveau sémantique sont à surmonter grâce au procès métaphorique soutenu par les parallélismes phonologiques et rythmiques et aboutissent à la création d'un nouveau sens : "Le pavillon en viande saignante" : "pavillon" et "viande" sont d'abord incompatibles, mais reliés par le parallélisme phonologique /vij/: /vj/; par leur juxtaposition les deux termes sont amalgamés dans une seule image de boucherie ("viande") et de bataille cruelle.

"la soie des mers" "[ la soie] des fleurs" : par deux fois, les qualités de la soie, être doux, reluisant et chaud, forment un tout inhabituel avec les qualités de la mer et des fleurs.

"fleurs arctiques" : la combinaison oxymorique unit la froideur morte, infertile et sans couleur de l'arctique et le symbole par excellence de la vie, du printemps et de la couleur, dans une image utopique qui surmonte cette "impossibilité" à l'instar du topique "désert florissant".

Le refrain esquisse l'image d'une bataille barbare sur le fond d'un paysage édénique. S'ajoutant au rôle unificateur des allitérations et des assonances pour ces deux tendances normalement incompatibles, il y a le niveau rythmique qui permet une différenciation significative supplémentaire :

Le pa villon en vi an de saignan te

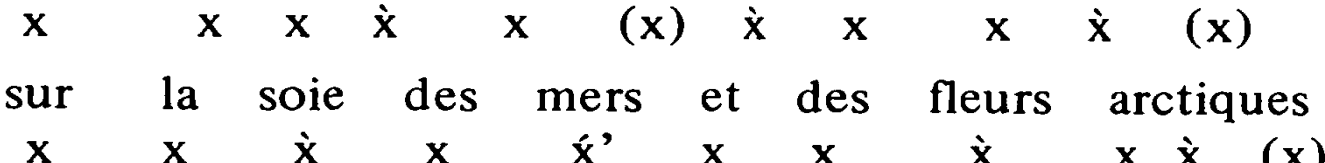

(elles n'ex istent pas)

$\mathrm{x}(\mathrm{x}) \quad \mathrm{x} \quad \mathrm{x} \quad \mathrm{x} \quad \mathrm{x}$

En établissant une unité et en même temps une différence sensible entre les deux vers féminins d'un nombre presque égal de syllabes (le premier sans césure, le deuxième scindé en deux parties isométriques, qui se répètent comme un écho dans l'hémistiche), c'est le rythme qui crée la liaison entre les éléments du refrain. Au niveau sémantique comme au niveau rythmique, il y a unité malgré les différences; dans une seule image sont amalgamés des éléments hétéroclites. Un mouvement "barbare" sous un pavillon rouge livre une bataille sanglante, mais une bataille qui doit être vue sur le fond d'un état utopique encore non-réalisé ("elles n'existent pas") mais déjà "vu" par le poète. Dans le refrain des éléments barbares, cruels et destructifs, se fondent dans la paix, la chaleur et la douceur d'un bonheur inconnu. 
L'élément destructif et sanglant s'insère sans difficultés dans l'isotopie traditionnelle et idéologique du titre "Barbare." Mais cette valorisation négative doit être mise très tôt en question par le lecteur qui est amené par les paragraphes I et III à considérer le refrain (II et IV) comme un progrès positif, en comparaison avec les "vieilles fanfares d'héroisme" et les "anciens assassins". Il s'agit, de toute évidence, d'une barbarie nouvelle, étrangère à cet héroïsme pathologique ("remis des") des entreprises militaires passées qui n'étaient qu'assassinats.

"Barbare" c'est un regard sur l'avenir qui, bien que réalisé dans le texte poétique, est toujours caractérisé par une grande distance temporelle ("bien après les jours et les saisons"), spatiale ("[ bien après ] les pays") et existentielle (" [ bien après ] les êtres"). Le hic et le nunc du présent par contre appartiennent en face de la "réalité" poétique déjà au passé ("vieilles", "anciens") bien qu'ils soient toujours actifs ("qui nous attaquent encore le coeur et la tête").

La perspective future du refrain n'est développée pleinement que dans la deuxième partie du texte (V - IX). La mise en relief du paragraphe V ("Douceurs!") marque son début et répond d'une façon programmatique au titre "Barbare."A la brutalité barbare se substitue un monde plein de douceurs et d'amour, au carnage et à la destruction un monde plein d'harmonie ("ô monde, ô musique!").

Comme dans la première partie, les mots qui pourraient appartenir à l'isotopie "barbare" sont par des contextes oxymoriques arrachés à leur limitation monosémique. Le feu destructif des "brasiers" est neutralisé par les "rafales de givre" et combiné dans une espèce de processus chimique qui réunit, sous une tension extrême, des éléments opposés. La deuxième moitié du paragraphe VI reprend ce processus, dans une image très complexe, en faisant allusion aux conditions extrêmes sous lesquelles le charbon se purifie en diamant. La répétition de "Douceurs" (V, deux fois en IX) rapproche cette cristallisation d'un processus d'affinage et d'amalgamation plein de tension et de voluptés.

Une synthèse semblable d'oppositions extrêmes (VIII : brasiersécumes; gouffres-astres; glaçons-astres) est appelée "musique" par synesthésie. Entre ces deux images complexes d'union créatrice du contradictoire et du destructif, est inséré un rappel de l'état présent qui précède la vision (VII) : "retraites" fait allusion à l'isotopie repoussée du militaire et les "vieilles flammes" aux anciennes formes de destruction.

Les moments négatifs du concept "Barbare" se sont brûlés et fondus dans le vertige d'une recréation chaotique dans la confusion des éléments (feu : brasiers, feux, carbonisé, astres; eau : pleuvant, givre, pluie, écumes, glaçons; air : rafales, vent; terre : coeur terrestre) - processus chimique qui aboutit à un nouveau monde ("ô monde"). De ce nouveau monde créé par le poète et son lecteur, fraternellement compris dans le même pronom ("carbonisé pour nous" en position marquée à la fin de la phrase) résulte l'harmonie inespérée d'un tel 
procédé "barbare", des "Douceurs" et de la musique. La vision s'élẻve d'une espèce de réaction chimique à une fête d'amour orgiastique au paragraphe IX. Les brèves exclamations introduites par "ô", le balbutiement anaphorique ternaire ("Et là ..."; "Et les larmes"; "- et la voix") rendent le rythme haletant et ne le laissent couler librement qu'à la fin du paragraphe :

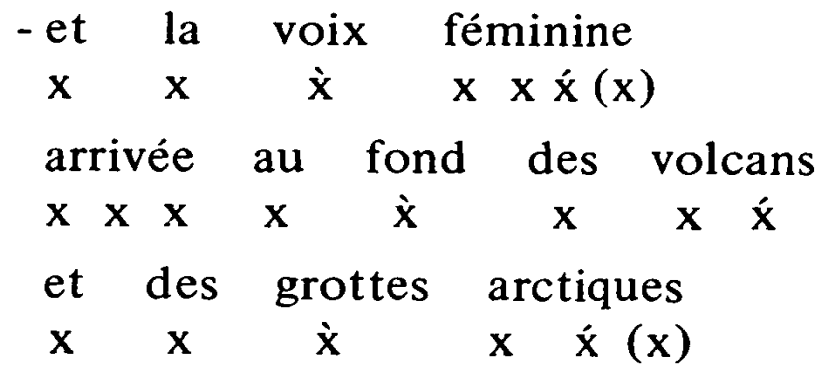

Le rythme et la syntaxe de ces lignes rappellent avec la rime identique ("arctique"), les allitérations $(/ \mathrm{v} /, / \mathrm{f} /)$ et les assonances $/ \mathrm{o} /$, la structure du refrain (sans sa parenthèse), repris en effet, bien que de manière fragmentaire, au paragraphe suivant. L'image utopique du refrain, caractérisée expressis verbis comme inexistante, est sous une autre forme, devenue réalité poétique. La reprise de refrain entier est superflue. La vision utopique s'est concrétisée dans l'amour humain ("formes", "sueurs", "chevelures", "yeux", "larmes blanches") (21), identifié à un mariage des contraires ("volcans" - "grottes arctiques") et inspiré par la capacité féminine de communication ("voix féminine').

"Barbare" nous fait suivre un changement de sens qui se distingue nettement du procédé utilisé par Jules Michelet cité plus haut, et que nous avions caractérisé comme une substitution de sens, au moyen d'un procédé métaphorique tronqué par une paraphrase conceptuelle. En modelant "Barbare" Rimbaud ne remplace pas une définition lexicale par une définition contraire, mais il fond le barbare dans le doux, le bruit dans la musique, le feu dans l'eau, le chaud dans le froid en une seule image complexe qui ne se laisse plus conceptualiser, mais quand même re-construire et interprèter. Une vision du monde "simple" qui ne voyait dans le barbare que le côté négatif, est élargie et mise en branle. L'incompatibilité sémantique force l'imagination du lecteur à surmonter ses préjugés, et à réaliser poétiquement les possibilités utopiques d'un amour créateur.

La référence du texte "Barbare" ne se trouve pas dans la référence de quelque mot isolé, par exemple dans le drapeau rouge des socialistes, dans un voyage de Rimbaud à Stockholm (juin 1877), voire dans les geysers d'Islande, mais cette référence se trouve dans la structure de l'image toute entière d'un certain développement historique et d'un futur utopique.

Voir les "larmes de lait" du stuprum "Obscur et froncé....". 
Le modèle de Rimbaud n'escamote pas, comme chez Michelet, le côté destructeur de son mouvement barbare, mais il détruit pour être constructif. Pour faire ressortir les éléments caractéristiques du modèle de Rimbaud, il serait utile de le comparer à d'autres modèles du "barbare" qui lui sont contemporains.

V - "Barbares" c'était, pendant tout le XIXème siècle, un des mots préférés pour désigner les "classes laborieuses et dangereuses" (22). Bien que cette désignation semblât tomber en désuétude, sous l'influence d'une ouvre comme celle de Michelet, les événements autour de la Commune de Paris faisaient revivre les vieux préjugés. L'exécution des otages, parmi eux l'archevêque de Paris, dans les derniers jours de la Commune, la responsabilité des incendies qui détruisirent des monuments historiques nationaux, tels que les Tuileries, rénovèrent l'image d'une populace barbare et iconoclaste, pas seulement chez les réactionnaires, mais aussi chez les libéraux (Flaubert) et même chez ceux qui faisaient jadis partie de la gauche (Leconte de Lisle, George Sand) (23).

A plusieurs endroits de ses Tableaux de Paris (1871) Théophile Gautier ne cache pas son indignation à l'égard des communards "barbares" qu'il compare aux "bêtes fauves" et aux "gorilles" (24). Mais pour un vrai poète de l'art pour l'art ces bassesses politiques sont indignes de sa plume. Un extrait de l'article "Les Barbares modernes" de Théophile Gautier, montre bien quel intérêt un tel écrivain pouvait porter à la réalité :

"Un groupe de communards prisonniers est poussé, sous une chaleur de plomb, vers le camp de Satory, au sud de Versailles: Nous regardions ces cavaliers de si grand style, regrettant qu'un peintre de génie ne se trouvât pas là, pour fixer d'un trait rapide ces belles lignes naturellement et naivement héroíques, et aussi pour noter les types non moins curieux des captifs, devenus des prisonniers barbares, Daces, Gètes, Hérules, Abares, comme on en voit dans les bas-reliefs des arcs de triomphe et les spirales des colonnes Trajanes. [...] Blouse, blaude, sayon, tunique, à cet état, se ressemblent fort; et les braies sont, dans la sculpture antique, le signe distinctif du barbare; [...] D'autres avaient garni leurs pieds meurtris de chiffons retenus par des cordelettes, qui leur donnaient un aspect de Philoctète dans son île, à faire rêver un sculpteur. Ce bout de haillon les rattachait à l'art grec. [...] Parmi ces prisonniers, il y avait quelques femmes, assises sur leurs articulations ployées, à la manière des figures égyptiennes dans les jugements funèbres, et vêtues de haillons terreux, mais donnant des plis superbes. [...]" (p. 241 et suiv.).

Pour un poète "subjectif", tel que le conçoit Rimbaud dans sa lettre du 13 mai, la réalité n'est qu'un réservoir d'images pittoresques

(22) L. Chevalier, Ouvr. cit., p. 595 et passim.

(23) P. Lidsky, Les Ecrivains contre la Commune, Maspéro, 1970.

(24) Th. Gautier, Tableaux de Paris 1871, dans Oeuvres complètes, réimpression de l'édition de Paris (1891), Genève, Slatkine, 1978, vol. III, p. 372. 
qui plaisent au public et qui font ressortir sa propre qualité d'artiste. Les hommes en haillons se figent en reliefs intemporels; les causes de la guerre civile, les espérances et les douleurs politiques des prisonniers qui ne sont rien de moins que les concitoyens de Gautier n'entrent pas en vue. En revanche, pour Rimbaud, ce n'est pas le côté décoratif du barbare qui est au centre de son intérêt, mais ce sont ses possibilités utopiques. De Fourier et de Michelet, il reprend l'idée d'un "affranchissement par l'amour", d'une société harmonieuse sans exploitation et aliénation. Gautier esthétise le "servage" (25) d'un point de vue désintéressé, parce que non touché en qualité de bourgeois et membre de la classe victorieuse. Rimbaud et son lecteur se meuvent au centre du procès barbare d'une métamorphose du monde vers un avenir positif.

Par ce trait, Rimbaud se distingue aussi des Poèmes barbares (1862 - 1878) de Leconte de Lisle. Après une jeunesse fouriériste, celui-ci s'est détourné de la politique et s'est réfugié dans un art pur et absolu. La barbarie de ses poèmes est nostalgique; il évoque ce qui est loin dans le temps et dans l'espace, la brutalité, le fanatisme, les passions de l'origine de l'humanité ou de ses survivances ataviques ou exotiques; il modèle ses images comme modèles contrastant avec notre civilisation qui est, elle, mûre pour le trépas dans une explosion apocalyptique sans Dieu, sans lendemain et purement destructive :

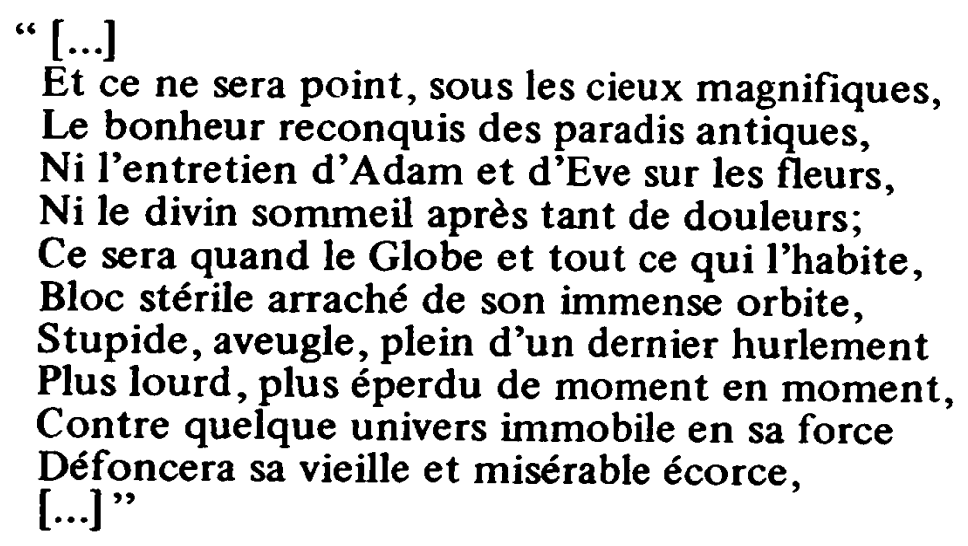

("Solvet seclum")

Rimbaud, de son côté, même après la répression de la Commune ne s'est pas résigné. La triste réalité n'inflige pas de démenti à ses convictions; au contraire, la certitude que l'harmonie universelle ne se fera que par un procédé sanglant, barbare, non pas en réconciliant les éléments sociaux, mais en les amalgamant, en les fondant dans une matière unique et inséparable par la force de l'amour.

Le fait qu'il ne s'agisse dans "Barbare" que d'une vision utopique, a favorisé le malentendu selon lequel le texte de Rimbaud ne serait que le produit d'une "fantaisie dictatoriale" caractérisée par une "irréalité

(25) "Du servage et de la haine" et "De l'affranchissement par l'amour" sont les titres des deux parties du Peuple de J. Michelet; voir note 13. 
sensuelle" pour laquelle "la comparaison avec la réalité serait inutile" (26). Le caractère essentiel de la poésie moderne échappe à une telle optique. Toute vision du monde sortant du rang de ce qui est communément admis, semble irréelle au gros des lecteurs. Mais, chaque image de la réalité est en quelque sorte irréelle : l'écart ontologique entre la réalité et le modèle reste toujours le même - à la seule différence près que les modèles "réalistes" sont "en arrière" des idéologies établies (c'est parce qu'on "re-connaît" ces visions du monde qu'on les "comprend") et que la poésie moderne est "en avant", construisant avec la matière idéologique des images anciennes, un nouveau modèle du monde.

(Université de Mannheim)

(26) H. Friedrich, Die Struktur der modernen Lyrik, Hamburg, 2 1967, p. 80-81. 


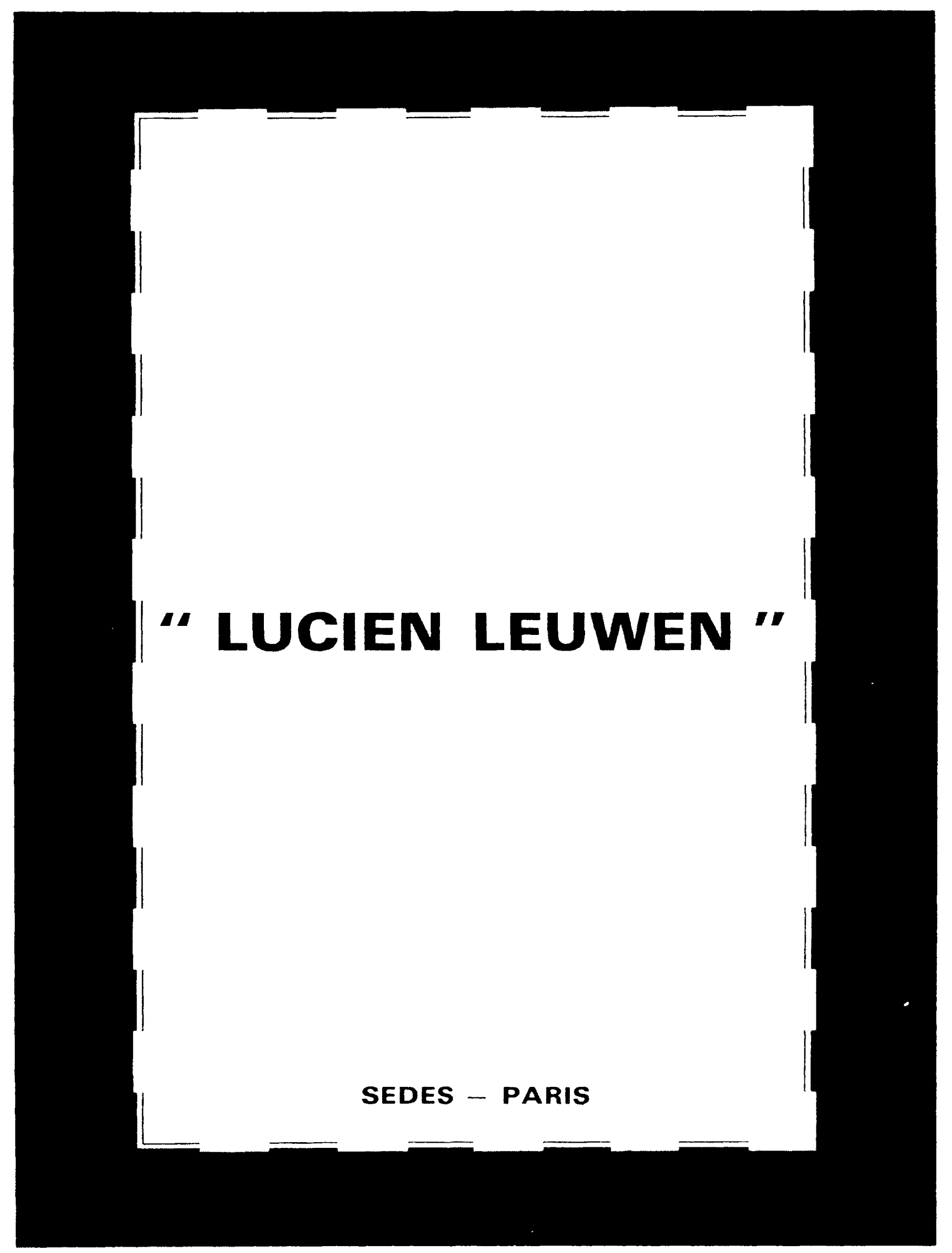

Un volume $(16 \times 24), 156$ pages, $82,00 \mathrm{~F}$ t.t.c. 